\title{
Buscando un por qué a los procesos de enseñanza y aprendizaje del clarinete en su etapa inicial
}

\section{Looking for a reason to teaching and learning the clarinet at an early stage}

\author{
Luis Adolfo Víquez Córdoba \\ Principal de clarinete de la Orquesta Sinfónica Municipal de Heredia \\ Profesor de la Cátedra de Clarinete, Escuela Municipal de Música de Paraíso \\ Costa Rica \\ laviquezcordoba@gmail.com
}

\begin{abstract}
Resumen: La formación instrumental ha tomado importancia dentro de la Educación Musical costarricense en los últimos tiempos, sobre todo con el auge de escuelas de música y academias en diversas comunidades a lo largo del país. El clarinete ha sido un instrumento de importancia en la vida musical de Costa Rica, desde la música militar hasta los ámbitos folclóricos y académicos. Tomando en cuenta los inicios de las actividades docentes instrumentales, en Costa Rica puede apreciarse que en el periodo de tiempo anterior a finales del siglo XIX muchas prácticas docentes se realizaban de forma empírica, situación que cambia posteriormente con la fundación de los primeros centros de enseñanza musical del pais.

Sin embargo, el enfoque dado a la educación del instrumento fue mayoritariamente orientado a la formación de músicos capaces de suplir las necesidades de los diferentes escenarios artísticos del pais, dejando de lado toda una serie de beneficios que la práctica instrumental puede dotar a las personas que se inician en ella. De este modo, por medio de los procesos de enseñanza y aprendizaje se pueden inculcar en las personas no solo destrezas musicales, sino habilidades para la vida, valores y actitudes que el o la estudiante, de no continuar con una carrera formal
\end{abstract}

\section{Introducción}

La Educación Musical en Costa Rica ha sido una disciplina que en los últimos años ha generado un constante interés por parte de diversos estudiosos del área, así como por personas que se sienten atraídas por el lenguaje de los sonidos y sus implicaciones dentro de la formación educativa de las poblaciones infantil y juvenil. Por un lado, es apreciable el gran esfuerzo que realiza el Ministerio de Educación Pública con el programa Ética, Estética y Ciudadanía con miras a fortalecer la formación artística como parte de la formación integral de los educandos. Fuera del ámbito escolar existen diversas instituciones de educación instrumental, las cuales cuentan con un currículo más específico dentro del aprendizaje musical como lo 
de música, podría aplicar en el futuro. Es así como las prácticas instrumentales del clarinete favorecen aspectos corporales como la postura, coordinación y respiración; afectivamente, por medio de la disciplina de la práctica individual o la ejecución de ensambles, la música permite a la población estudiantil desarrollar actitudes como el trabajo en equipo, la tolerancia $y$ el respeto, entre otros valores que sin duda colaborarán para la formación de los seres humanos del mañana.

Palabras clave: Educación musical, enseñanza del clarinete, educación instrumental en costa rica, filosofía de la educación musical.

\begin{abstract}
The importance of Instrumental Education in Costa Rica has been raised from many years ago, especially since the recent foundation of new schools of music and private academies in many comunities. As a musical instrument, the clarinet has been important in the history of music in the country from the army bands in the past to the recent groups of folk and classical music. With the musical instruction of the clarinet the students may learn not only musical skills but many habilities for their future life as values and attitudes. Playing the clarinet can improve physical functions as breathing, coordination and body positioning. In the other hand by individual practicing and ensemble performing the student can learn attitudes like hardworking, tolerance and respect to the people.
\end{abstract}

Key words: Music education, clarinet teaching, instrumental education in Costa Rica, phylosopy of music education son los conservatorios, Etapas Básicas de Música, escuelas de música, programas de orquesta y diversas academias privadas en las cuales la persona interesada acude en búsqueda de una instrucción más detallada en el área de la ejecución instrumental y el canto.

El clarinete -instrumento de la familia de viento-madera $\square$ ha estado presente en muchas facetas de la idiosincrasia del país, viéndose su uso desde la antigua milicia hasta las agrupaciones folclóricas y de música académica. La iniciación musical del clarinete no solo propicia los conocimientos básicos para la ejecución del mismo, sino que promueve una variedad de destrezas que con el paso del tiempo se desarrollarán para proveer a los estudiantes diversas habilidades para su vida futura.

Por esta razón, el presente artículo pretende ir más allá de las implicaciones técnico-musicales del instrumento, ya que busca ver cómo se pueden reforzar diversas características sociales, físicas y afectivas por medio de los diferentes aspectos pedagógicos de la técnica, la práctica en ensamble y la ejecución musical.

\section{Un vistazo al pasado y presente de la formación instrumental en Costa Rica}

Dentro de la historia de la música y del arte, la práctica instrumental ha ocupado desde siempre un lugar privilegiado, en el cual se sitúa en la élite del mundo musical a la persona intérprete, y mayoritariamente la perspectiva de la enseñanza musical se ha visto inclinada a atender las necesidades de esta ${ }^{1}$, lo cual se refleja en la gran cantidad de escritos sobre técnica e interpretación de los diferentes instrumentos musicales y la voz humana ${ }^{2}$.

La cultura europea, cuyo alcance se difundió por el resto del mundo como modelo de instrucción musical, ha implantado desde la formación de los primeros 
conservatorios un modelo de enseñanza basado en la competitividad y la exigencia. Con ello se ha estimulado una formación musical en las personas desde sus primeros años de vida, lo cual dentro del contexto de Occidente ha mostrado logros notables al ver cómo se egresan de los conservatorios y universidades músicos con un alto calibre técnico e interpretativo. Ponce de León y Lago (2009) explican que el proceso de maduración dentro de un conservatorio es de larga duración, dado a que los estudios se comienzan desde la infancia, y que difícilmente se puede conseguir en una etapa adulta sin haber tenido una formación previa la cual le haya suministrado las capacidades necesarias para hacer una carrera de música. El autor y la autora agregan que al finalizar sus estudios existe un perfil de salida en el cual el discente se egresa con un conocimiento mínimo de las diferentes áreas de estudio de la institución.

Durante el siglo XX y hasta estos días se ha tratado de implementar este modelo de conservatorio en países latinoamericanos. En el caso de Costa Rica, con la aparición de las primeras instituciones musicales de enseñanza y con la llegada de músicos extranjeros $\square$ quienes ejercieron labores educativas en el país $\square$, se ha tratado de seguir un patrón de formación de conservatorio, donde el centro de la formación del estudiantado ha sido el dominio técnico e interpretativo de su instrumento y paralelo a ello se le ofrecen cursos de rudimentos teóricos musicales y prácticas en ensambles de diversa índole.

En el libro "De las fanfarrias a las salas de concierto", escrito por la intérprete e historiadora María Clara Vargas (2004), puede apreciarse detalladamente cómo la instrucción musical en Costa Rica ha pasado por diferentes etapas. Iniciando desde la época colonial con los primeros frailes y maestros de capillas pasando por las fanfarrias y bandas de la milicia. Sumado a esto la autora menciona músicos provenientes del exterior y artistas que se presentaron en San José tras la creación del Teatro
Nacional, quienes también impartieron clases musicales.

Sin embargo, de acuerdo con Vargas (2004), la historia de la pedagogía instrumental costarricense ha tenido un trasfondo importante con la formación musical que músicos nacionales tuvieron en el extranjero a finales del siglo XIX y principios del siglo $\mathrm{XX}$, quienes al regresar al país formaron sociedades de concierto, asociaciones musicales y escuelas de música. Tres instituciones importantes fueron la Escuela Nacional de Música, de 1890 a 1894, posteriormente la Escuela de Música Santa Cecilia (1894 a 1956) y por último el Conservatorio de Música y Declamación fundado en 1915. Profesores como Roberto Cantillano, Julio Fonseca, Alejandro Monestel y José Joaquín Vargas Calvo formaron parte de las distintas cátedras de dichas instituciones.

Rosabal (2009) señala que a partir del siglo XX se da en Costa Rica el florecimiento de instituciones de instrucción musical formal como el Conservatorio Nacional de Música en $1942 \square$ el cual se incorpora a la Universidad de Costa Rica en $1968 \square$ y el Programa Juvenil de la Orquesta Sinfónica Nacional en $1971^{3}$. El autor también indica el surgimiento $\square$ en la década de los ochenta $\square$ de los programas de las Escuelas Municipales de Música, formadas conjuntamente por la colaboración el Ministerio de Educación Pública y el Ministerio de Cultura, así como hace referencia también al recién creado Sistema Nacional de Educación Musical, el cual propone un modelo de formación musical y extensión social en comunidades alejadas del país ${ }^{4}$.

En todo este tiempo de formación musical, los grupos infantiles y juveniles se han visto iniciados en sus instrumentos de múltiples formas. Específicamente en el caso del clarinete, con frecuencia no se tuvo un parámetro teórico adecuado y generalizado acerca del proceso de enseñanza-aprendizaje del instrumento, lo cual ha llevado a tener criterios diferidos con 
respecto a esta incógnita. El uso de métodos progresivos ha imperado mayoritariamente a lo largo de la formación musical de los clarinetistas en Costa Rica y se pueden contar muchos textos sugeridos por docentes e instituciones, sin embargo no ha existido un criterio unificado con respecto a objetivos de la educación clarinetística en la población infantil escolar y personas jóvenes.

El uso de los métodos paso a paso ha sido la forma pionera de educar a la población estudiantil, a partir de un orden de procedimientos cual si fuera una fórmula química para obtener un resultado esperado. Esto quiere decir que la metodología busca seguir reglas y patrones fijos con los cuales el personal docente sabe o espera qué rumbo va a tomar la instrucción del estudiantado. Esta metodología si bien ordena la consecución de objetivos, se aparta en muchas ocasiones de la realidad e intereses del educando. Se produce una descontextualización en la enseñanza, dado a que el procedimiento en sí mismo deja de lado el entorno en el cual se desenvuelve el estudiantado y sus necesidades.

Generen o no discusiones, estas metodologías poseen un enfoque conductista. De acuerdo con Méndez (2008), el conductismo aboga a una educación que considera formar hábitos, reforzamiento de destrezas y extinción de actitudes no deseadas en el alumnado. Desde esta perspectiva metodológica, los procesos de enseñanza y aprendizaje se convierten en una transmisión de conocimiento ${ }^{5}$ en la cual el o la docente se convierte en figura de autoridad y el énfasis es de mero rendimiento académico, dejando de lado la integridad de la persona.

Calderón (2008) expone que en el conductismo se evalúan solo las conductas que son observables, por lo cual la población estudiantil se encuentra en una situación pedagógica de estímulo-respuesta, se vuelve pasiva y replica el conocimiento, no lo produce.

Es notorio ante este planteamiento que la formación clarinetística costarricense se puede etiquetar como "procedimental". Esto refiere a la forma en que se evalúa, ya que con frecuencia en un recital o examen solamente se miden los resultados in situ y se deja de lado todo un proceso cognitivo y afectivo previo, por lo que esta situación da hincapié a una reflexión acerca del rol que asume el personal docente en el proceso y el grado de compromiso que este adquiere con su trabajo ${ }^{6}$.

Formar niños, niñas y jóvenes, sobre todo en las disciplinas instrumentales, se convierte en un proceso complejo, ya que implica un bagaje pedagógico adecuado y un alto grado de vocación por parte del profesorado. A menudo no se cumplen estas expectativas ya que en ocasiones son contratados excelentes instrumentistas, pero que no poseen las herramientas pedagógicas necesarias para la enseñanza del instrumento. La clarinetista y pedagoga costarricense Yamileth Pérez (2007) explica que la obligación de la y el docente es contribuir a la formación integral del estudiantado y no limitarse a dar información veraz y detallada, lo cual perjudica a la persona estudiante situándole como objeto híbrido entre máquina-humano, en vez de personas con el compromiso de replantear su presente y edificar su fututo. Con esto puede apreciarse que el formador musical se ha visto dentro de una relación vertical de profesor-estudiante donde el aprendizaje no se crea ni se construye, sino que se replica.

Hablando didácticamente, los métodos y libros utilizados en los procesos de enseñanza y aprendizaje del clarinete derivan mayoritariamente del idioma inglés, lo cual presenta una limitación tanto para la población estudiantil como para el personal docente. Muchas veces se utilizan copias realizadas sobre otras reproducciones rayadas o con escritos y, aunque no parezca relevante, la presentación del material didáctico no brinda mayor interés para que el alumnado se entusiasme con este. También puede darse que las condiciones físicas y ambientales de los lugares 
donde se realiza la enseñanza no son las adecuadas para trabajar con poblaciones infantiles o juveniles por múltiples razones de ubicación o infraestructura ${ }^{7}$.

Otra problemática por la cual no existe una metodología objetiva con respecto a la formación clarinetística en su etapa inicial es la falta de claridad en los objetivos de la educación instrumental de las instituciones en las que se imparten los diferentes instrumentos musicales. Se ha esperado siempre que el resultado de la formación musical se incline a graduar a un concertista, lejos de facilitar las destrezas básicas a una persona íntegra, amante de su sociedad, de su cultura y con compromiso de trabajo. Se ve a cada estudiante como un futuro instrumentista y no como un ser partícipe de su comunidad que se encuentra en formación y que se haya ligado a un contexto que delimita su proceso educativo y por el cual debe trabajar a futuro por mejorarlo.

Técnicamente no existe certeza acerca de qué se pretende enseñar en los estudiantes. Se ha tratado de abarcar cuantas destrezas sean posibles sin tener idea de que no se han establecido bases técnicas imprescindibles tales como la postura, la respiración y la embocadura. El problema ha radicado en que se pretende que el estudiantado toque obras que sobrepasan sus habilidades iniciales y es frecuente ver cómo los objetivos de los ensambles instrumentales no van ligados a las posibilidades técnicas del instrumentista. Con esto se da que los estudiantes aprenden malas prácticas - popularmente llamadas "mañas" $\square$ con tal de rendir con las partes que les piden en banda, orquesta o música de cámara.

En términos generales en la pedagogía instrumental de Costa Rica ha sido difícil definir el verdadero propósito de la formación musical. Se ha encasillado el aprendizaje a la producción de instrumentistas profesionales y no a la formación de personas que aprovechen los procesos de enseñanza y aprendizaje de la música para desarrollar sus capacidades intelectuales de una mejor forma. Por ello es necesario definir cómo el personal docente puede contribuir a esto desde las aulas y cómo por medio de las distintas implicaciones técnicas e interpretativas de la formación clarinetística se puede colaborar a un mejor desarrollo intelectual y afectivo de la población estudiantil.

La educación en todos sus ámbitos debe abordar grandes retos en la Costa Rica de hoy en día. Como lo expresa la educadora costarricense María Eugenia Dengo (2002), los procesos educativos son la herramienta para preservar la identidad del país y la educación costarricense ha impreso rasgos propios de la idiosincrasia costarricense como el apego a la libertad, la independencia, la soberanía y un estilo de vida democrático. La autora insta a pensar que "la educación sirve a las finalidades y los paradigmas que la sociedad le señale" (p. 29).

Considérese esta reflexión: ¿por qué no orientar la educación instrumental costarricense hacia un eje más axiológico? El docente puede dar no solamente una formación musical de alta calidad sino infundir valores y actitudes que sean significativos en la vida del estudiantado como el respeto, el amor, la tolerancia, el empeño y trabajo. De acuerdo con Calderón (2008) los valores no se apropian cual si fueran dogmas, sino que se vivencian en la conciencia de la humanidad a lo largo del tiempo.

\section{La situación de las condiciones de aprendizaje en Costa Rica}

Como se mencionó antes, en la formación instrumental clarinetística del país ha imperado un modelo de conservatorio heredado de las tendencias que muchos docentes nacionales trajeron, quienes desde inicios del siglo XX viajaron a tierras extranjeras a realizar su formación musical. Asimismo, debe recordarse que se contó con la llegada de docentes provenientes de otras latitudes 
quienes de forma momentánea o permanente se establecieron en tierras costarricenses y ofrecieron sus servicios musicales y de docencia (Vargas, 2004).

Pérez (2007, p.178) menciona que en la educación musical costarricense "se instauró una forma de enseñanza de predominio occidental, que ha abarcado esencialmente tres ejes temáticos: el estudio técnico del instrumento, el adiestramiento rítmico y auditivo y el estudio sobre historia y teoría musical provenientes de Europa". Al hablar de un modelo de conservatorio imperante en la formación clarinetística del país, debe hacerse mención a una serie de características que esta ha tenido:

Verticalidad en la relación entre sujeto docente y la población estudiantil: El personal docente se ubica en la cúspide de esta relación, ya que es el elemento imperante en la misma. Visto desde el enfoque conductista, el profesorado es quien brinda la información y define las reglas y los contenidos del aprendizaje. El estudiantado se sitúa subordinado a los y las docentes. Actúa de forma pasiva y se concentra en reproducir a cabalidad las instrucciones brindadas por la persona mentora.

Predominio del método o libro de aprendizaje como orientador del proceso: En la formación clarinetística costarricense por muchos años se ha utilizado una alta gama de métodos, libros y folletos con los cuales se ha "unificado" el currículo estudiantil. Por lo tanto, el rumbo de los procesos de enseñanza aprendizaje lo lidera el material didáctico. Los objetivos del proceso pedagógico del clarinete son determinados por una serie de competencias que se espera que el estudiantado alcance al concluir cada libro o folleto suministrado por los y las docentes.

Evaluación orientada exclusivamente a resultados finales observables: La población estudiantil se ve medido de acuerdo a la consecución de sus objetivos presentados en un único momento final, tales como un recital público o un examen de instrumento frente a un comité evaluador denominado jurado. En algunas ocasiones el resultado de este momento de evaluación final determina la continuidad de las personas en los procesos de enseñanza y aprendizaje.

Otro ejemplo de situación de enseñanza y aprendizaje del clarinete en Costa Rica es que los objetivos y competencias deseadas han sido definidos de acuerdo a las necesidades y estándares de las actividades de una institución y se ha dejado de lado los intereses de la persona en sí. En el caso de los ensambles instrumentales es notorio el nivel de exigencia que se tiene hacia el estudiantado de clarinete, ya que es un instrumento protagonista en las bandas y orquestas sinfónicas. En las bandas, los clarinetes forman el cuerpo de la cuerda melódica del ensamble, por lo cual tienen vital importancia en el mismo; por otra parte, en las orquestas existe mayor protagonismo en solos o pasajes de las secciones de vientos maderas.

Con todo lo anterior entiéndase que las demandas de los ensambles instrumentales han favorecido el acelerar etapas y procesos para los cuales el clarinetista no está en condiciones técnicas o simplemente no ha alcanzado un nivel de madurez musical adecuado para cierto tipo de obras. Esto implica que en muchas orquestas y bandas comunales o de escuelas de música se exige al alumnado un repertorio que no puede tocar. Notoriamente esto puede llegar a producir:

Problemas técnicos de embocadura, articulación, respiración, postura general del clarinete y de las manos al colocarlas sobre el instrumento. Esto lo acrecienta la presencia de pasajes veloces o incómodos en los cuales la población estudiantil tiende a ejercer presiones indebidas con el instrumento y se ve automáticamente obligado a descuidar principios técnicos importantes con tal de aproximarse al contenido musical que aún no puede tocar.

Vacíos de lectoescritura y teoría musical. Muchas veces la lectura musical se vuelve viciosa y muchos ritmos y pasajes se aprenden por imitación y no 
son interiorizados por el grupo estudiantil. Puede que sea capaz de tocarlos correctamente en una obra determinada, pero va a encontrar problemas al enfrentarlos en una pieza musical nueva.

Sentimientos de baja autoestima y desmotivación. Algunos instrumentos musicales cuentan con mayor facilidad en determinados pasajes musicales que el clarinete. Cuando la persona que aprende no está lista para enfrentar determinados trechos, al ver que en el ensamble muchos de sus pares sí lo logran, este tiende a sentirse mal pues su rendimiento no es similar al de los otros. Esto genera desmotivación e incluso puede ser causante de que algún o alguna estudiante decida separarse del aprendizaje de su instrumento.

En fin, a lo largo de décadas las instituciones musicales han tenido expectativas específicas con respecto a lo que desean de sus estudiantes, disímiles con la realidad de las personas. Con esto se puede concluir que estas instituciones desean graduar músicos concertistas, solistas, músicos hábiles para la música de cámara, integrantes de orquesta, banda o conjunto de música popular. Estas expectativas se acrecientan en instituciones musicales de menor rango como las escuelas de música y academias privadas en las cuales el enfoque educativo se orienta a la formación de instrumentistas y se deja de lado la parte humana de la formación clarinetística.

\section{Beneficios y retos de los procesos de enseñanza y aprendizaje del clarinete}

Numerosos estudiosos (Lacárcel, 2003; Rosabal 2010) han demostrado que la actividad musical trae consigo beneficios para el desarrollo mental, social, afectivo y educativo del ser humano. Estos autores han establecido la importancia que la educación y práctica musical han tenido especialmente en las etapas de la niñez y adolescencia.
La práctica instrumental del clarinete trae en sí misma una serie de beneficios para el ser humano, desde el bienestar físico hasta el desarrollo socioafectivo y psicomotor. Para reflexionar sobre estos aspectos se realizaron entrevistas a cinco docentes del clarinete distinguidos en el ámbito nacional y latinoamericano. Carmen Small (Instituto Nacional de la Música de Panamá), Sergio Fernando Reyes (Conservatorio Nacional de Música de Guatemala) y Krista Helfenberger (2010, comunicación personal, Orquesta Sinfónica de Ribeirao Preto, Brasil). Así mismo, se tuvo el aporte de las clarinetistas costarricenses Rocío Maireno y Ana Pamela Goyenaga (2010, comunicación personal), ambas instructoras del Sistema Nacional de Educación Musical, quienes llevan una larga trayectoria como docentes del instrumento, es así como la información obtenida mediante dichas entrevistas permite ordenar los datos en aportes físicos y aportes socioafectivos, de la siguiente manera:

\subsection{Aportes físicos que brinda la práctica del clarinete a los niños y jóvenes}

Con la práctica del clarinete se ejercitan muchos aspectos del cuerpo humano. Primero, el tener un control sobre la respiración le crea a la persona hábitos correctos a la hora de respirar y le insita a tener cuidados con su sistema respiratorio. Desde el punto de vista de la postura el clarinete aporta nociones importantes de ergonomía para todo tipo de personas, ya que su práctica involucra concentrarse en colocar de forma correcta las extremidades, tronco y cabeza a la hora de practicar con el instrumento o tocarlo en público. Por otra parte, si la práctica instrumental se hace con disfrute y relajación puede colaborar notablemente a bajar los niveles de tensión y estrés muscular. Asimismo, el clarinete contribuye a mejorar la motora fina debido a la sensibilidad que se debe tener al 
momento de movilizar los dedos y coordinarlos a la hora de ejecutar determinados pasajes. Otro ejemplo de motora fina es la colocación de los labios y la lengua, ya que muchas veces son sensaciones abstractas puesto que es imposible verlos completamente.

De las personas entrevistadas, Rocío Maireno mencionó la coordinación como uno de los beneficios de la práctica del clarinete más allá de lo musical. Por su parte Krista Helfenberger (2010) menciona que el clarinete puede ayudar a cada estudiante de varias maneras a respirar correctamente, desarrollar la habilidad motora fina y tener control mental sobre sus movimientos corporales.

\subsection{Aportes de la práctica del clarinete a nivel mental y socioafectivo en la persona}

Mejoras en la concentración y control emocional fueron parte de los aportes que consideran las personas entrevistadas con respecto a los procesos de enseñanza y aprendizaje del clarinete. La profesora Helfenberger valora que por medio de la ejecución del fraseo, el o la alumna puede adquirir sensibilidad hacia el mundo que lo rodea ${ }^{8}$. Por su parte, Small menciona que los y las estudiantes "cuando grandes, si no culminan la carrera (estudios instrumentales), son más sociables o humanos, por medio de la sensibilidad artística”. La adquisición de la disciplina es un pilar con respecto a los aportes que la práctica clarinetística brinda al alumnado, ya sea por medio del estudio individual que se debe realizar fuera de clase o por los ensayos de ensamble instrumental, donde tener una actitud de respeto y seriedad es indispensable para el desarrollo correcto de la lección.

Dentro del ámbito del desarrollo afectivo y axiológico la práctica del clarinete suma numerosos aportes entre los cuales debe hacerse mención:
Con la práctica diaria, el estudiantado hace conciencia acerca de la importancia del trabajo tesonero como consecución de objetivos. El alumnado debe interiorizar que en la vida todo esfuerzo se ve recompensado de forma satisfactoria.

La práctica de ensamble (dígase coro de clarinetes, música de cámara, orquesta o banda) coadyuva a que el discente valore la importancia del trabajo en equipo. Valores como el respeto hacia los demás y la colaboración se manifiestan en estas prácticas. Asimismo, en un ensamble la población estudiantil aprende a ser tolerante y a aceptar la diversidad.

Al hacer música, el alumnado se ve instado a valorar la cultura y el patrimonio musical del contexto en el que vive. La población estudiantil adquiere conciencia que la música refleja la identidad de su comunidad y que es importante rescatarla ante las nuevas corrientes que buscan tener un mundo globalizado donde el ámbito local pierde fuerza.

En concordancia con las personas entrevistadas la práctica del clarinete dota de disciplina a los y las estudiantes. Mediante ella y sumando constancia y compromiso, la persona discente está ejercitando desde joven competencias que son comunes en la vida adulta.

\section{La pedagogía del clarinete en la sociedad actual. El rol del cuerpo docente}

En las entrevistas realizadas se consultó a los y las docentes sobre cómo poder ayudar a la inserción de discentes en la sociedad actual por medio de los procesos de enseñanza-aprendizaje del clarinete y el rol que debe asumir el o la docente ante las necesidades educativas instrumentales de hoy en día. Luego de ver los beneficios antes descritos ha llegado la hora de determinar los retos que el docente y la docencia deben asumir para hacer de su profesión un 
quehacer que brinde una enseñanza significativa a sus estudiantes.

Dentro de una clase, el maestro o la maestra pueden propiciar un ambiente cálido, en el cual la motivación y la confianza formen parte del incentivo que la población estudiantil requiere para seguir avante con su formación. De acuerdo con Maireno (2010, comunicación personal) el maestro o maestra de instrumento debe dejarse ver como un facilitador y no como un profesor, siendo una guía para el estudiante.

Es necesario en este punto señalar diversas características deseables del personal docente del clarinete que se necesita en estos nuevos tiempos. Primero no solo tener una importante trayectoria o vida artística favorable, ni siquiera ser un individuo dotado de perfección técnica y musical. El amor por la docencia en todos sus niveles se hace fundamental, ya que de esto depende el entusiasmo que el o la docente va a tener y su disposición para facilitar el conocimiento a sus alumnos o alumnas. Por otra parte, el profesorado debe ser un ser tolerante y aceptar que existe una numerosa diversidad de estudiantes y estilos de aprendizaje dentro de la enseñanza instrumental. Rosabal (2008) explica que:

Atender los estilos de aprendizaje de uno mismo, y más importante, de nuestros estudiantes, implica, por un lado, que adoptamos una actitud respetuosa y de aceptación de la diversidad de formas que las personas tienen para aprehender el mundo, desenvolverse en él, y relacionarse con otros. Por otra parte, constituye una decisión que abre ilimitadas posibilidades para el desarrollo exitoso y gozoso del estudio y la ejecución instrumental de uno mismo y de quienes confían en nosotros como mentores o educadores musicales.

Respetando el estilo de aprendizaje de la población estudiantil, el profesorado debe ser creativo y abierto a las distintas posibilidades y estrategias de mediación a las que se tenga que enfrentar. Debe tener claro que las habilidades técnicas e interpretativas son construidas junto con la y el estudiante, sus intereses y su contexto, además de buscar un estímulo al libre pensamiento, la construcción de desconocimientos y la adquisición de aprendizajes significativos (Carballo, 2000).

Además los y las docentes deben estar en constante actualización, estar anuentes a hacer investigación en su campo $\mathrm{y}$ tener roce con las últimas tendencias sobre pedagogía, técnica y estética de ejecución y combatir en todo momento el conformismo. Lamentablemente en el país ha existido mucha resignación a buscar las cosas y lograr lo posible con el menor esfuerzo. El conformismo y la mediocridad han estado presentes en algunos docentes e instrumentistas a nivel nacional, con ello se mantienen firmes con metodologías obsoletas y no se aventuran a investigar acerca de las nuevas tendencias pedagógicas o a los intereses de sus estudiantes. Se cae en lo que Carvajal (2010) menciona de una permanencia en una sociedad donde son características la apatía y el conformismo del "labriego sencillo".

Los procesos de enseñanza y aprendizaje del clarinete brindan altos aportes a la sociedad puesto que mediante esta práctica instrumental se construyen y refuerzan valores y actitudes en el individuo, también se contribuye a una mejor orientación de la sociedad ya que la población estudiantil no es ajena a la misma. Por ello es que desde las aulas, docentes y estudiantes se encuentran construyendo desde ya la sociedad del mañana. Por medio de la participación musical en comunidad, las y los alumnos de clarinete pueden sentirse importantes en el beneficio de la misma por medio de acciones concretas tales como recitales en ciudades alejadas, conciertos en cárceles, hospitales, asilos o escuelas, donde el estudiantado puede lograr sensibilizarse ante el entorno que le rodea.

Krista Helfenberger (2010, comunicación personal) menciona

Por medio de la enseñanza se modifica sin duda una sociedad, para bien o para mal. El rol del (la) profesor(a) es de extrema importancia. Por eso, en primer lugar estos profesionales (en cualquier área) deben estar muy bien preparados y sobre todo tener 
vocación para esa tarea y conciencia de su responsabilidad. En la enseñanza de la música se tiene la ventaja de poder trabajar con grupos pequeños de estudiantes. En la enseñanza de los instrumentos se trabaja tradicionalmente de manera individual. Por lo tanto, el profesor puede percibir muy bien las características de cada alumno, no solo en cuanto a sus habilidades para la música sino también de su potencial en general. En un trabajo donde existe una relación tan directa entre el profesor y el alumno, se crean lazos fuertes y favorables, o en otras ocasiones, no tan favorables. El profesor tiene que ser sensible a esta enorme responsabilidad servir de guía para que el alumno se desarrolle globalmente.

La lección de clarinete, de acuerdo al punto de vista de la docente, es un momento clave de cercanía con el estudiante debido a la atención directa y personalizada que tiene el y la estudiante con su docente. Es por esto que el ambiente que se propicie será clave para dar fluidez al proceso educativo. La clase es un momento único y el maestro o maestra de clarinete tiene en sus manos un espacio para dotar a sus estudiantes no solo de conocimiento musical, sino de habilidades diversas para la cotidianeidad a la que se tendrán que enfrentar en los próximos años.

\section{Reflexión final}

Los procesos de enseñanza y aprendizaje de la música y del clarinete no solo consisten en un tipo de instrucción metódica cuyo fin es lograr un dominio técnico e interpretativo de un instrumento. Va mucho más allá. La actividad musical brinda un sinnúmero de beneficios y dependiendo del contexto educativo en el cual se desarrollen estos procesos habrá más resultados positivos que ayudarán a la persona a desarrollar destrezas y habilidades aplicables en su vida futura.

Quien ejerza la docencia del clarinete debe orientar enseñanzas hacia un norte común en el cual las potencialidades de la persona se desarrollen al máximo. Un norte en el cual imperen los valores y las buenas actitudes dentro de la formación de los discentes y en el cual se aprenda que la vida consiste en un aprendizaje permanente y continuo. Por ello es que docentes, padres de familia y sociedad deben reflexionar acerca de cuáles aspectos de la enseñanza pueden ser más significativos para el estudiantado costarricense con tal de dar un eje axiológico a los procesos educativos. Por medio de la educación en todos sus aspectos se puede contribuir al progreso de las personas y por ende se obtendrá una sociedad mejor.

Tradicionalmente la enseñanza instrumental se ha orientado en tecnicismos, virtuosismos o dominios de vasta cantidad de repertorio y autores. Es necesario ir pensado más allá del dominio perfeccionista del instrumento y es indispensable un arduo trabajo que ayude a orientar la educación instrumental de las regiones latinoamericanas hacia un pensamiento más consciente y funcional en pro del enriquecimiento integral del ser humano y la sociedad de hoy en día.

\section{Notas}

$1 \quad$ En su artículo "Necesidades y orientación en los conservatorios profesionales de música", Luis Ponce de León y Pilar Lago, ambos pedagogos españoles, expresan que "la normativa establece que el estudio de un instrumento es el <<eje vertebrado $>>$ de las enseñanzas de música ya que se trata de la materia que acompañará al alumno a lo largo de todas sus enseñanzas elementales y profesionales" (Ponce de León y Lago, 2009: 3).

2 Ejemplos destacados de estos escritos son el Método de Flauta de Quantz, el célebre tratado violinístico de Leopold Mozart, el Método de Canto de Vaccai y una notable lista de métodos de ejecución del clarinete como los de Lefebvre, Klosé, Dangain y Bonade.

3 Hoy en día en convenio con la Universidad Estatal a Distancia, el Instituto Nacional de Música (Antiguo Programa Juvenil) ofrece el grado de Bachillerato y Licenciatura con concentración en Estudios Instrumentales.

4 Aclárese que en las Escuelas Municipales de Música y en las Etapas Básicas de la Universidad de Costa Rica se obtiene un grado de técnico medio en música al finalizarla. Dicha titulación 
es reconocida dentro escalafón profesional del Ministerio de Educación Pública. Pionera en este tema fue la Escuela Municipal de Música de Paraíso de Cartago, fundada en 1983 como la primera escuela municipal de música del país. Sin embargo, se debe agregar a manera de comentario personal que son pocos los graduados por estas instituciones, ya que ofrecen un proceso que incluye varios años de formación y muchos estudiantes antes de finalizar sus estudios en las mismas ingresan a los programas de universidades estatales y otras instituciones de formación superior

La autora agrega que a menudo este aprendizaje no es funcional ya que el estudiante aprende algo que no está entendiendo.

Las pruebas de instrumento, por lo general, se presentan frente a un jurado individualmente. $\mathrm{Al}$ estudiantado le es solicitado la ejecución de ejercicios técnicos, extractos orquestales u obras del repertorio de su instrumento y al final los profesores calificadores emiten una calificación, acompañada de sus respectivas observaciones. Entre las principales problemáticas están la falta de espacio y la carencia de condiciones acústicas donde de pueda suprimir el ruido exterior. Esto sin contar que muchas agrupaciones musicales comunales simplemente cuentan con un salón grande donde convergen los docentes de los diferentes instrumentos.

Helfenberger se refiere al uso del fraseo para contrastar diversas sonoridades musicales y estados anímicos.

\section{Referencias bibliográficas}

Calderón, K. (2008). La vida que enseña. Nociones de educación permanente. San José, Costa Rica: Editorial UNED.

Carballo, S. (2000). Retos de la maestra y del maestro del siglo XXI. Revista Educación, No. 24. San José: Costa Rica.

Carvajal, M. (2010). Cinco temas en busca de un pensador. Una lectura desde la modernidad-posmodernidad. Istmo: Revista Virtual de estudios literarios y culturales centroamericanos, No. 20. San José, Costa Rica. Recuperado de http://collaborations. denison.edu/istmo/n20/articulos/9carvajal_maria_form.pdf
Dengo, M. (2002). Nuevos paradigmas para la educación. San José, Costa Rica: Editorial UNED.

acárcel, J. (2003) Psicología de la música y emoción musical. Revista Education, no 20-21. Recuperado de: www.um.es/ ojs/educatio/article/view/138/122

Méndez, Z. (2008) Aprendizaje y cognición. San José, Costa Rica: Editorial UNED.

Pérez, Y. (2007) Análisis crítico del quehacer docente de los músicos. Revista Actualidades Investigativas de Educación, 7(1). San José, Costa Rica. Recuperado de http://revista. inie.ucr.ac.cr/articulos/1-2007/archivos/musicos.pdf

Ponce de León, L., y Lago, P. (2009) Necesidades y orientación en los conservatorios profesionales de música. Revista Electrónica LEEME, No. 24. Recuperado de http://musica.rediris. es/leeme/revista/ponce\&lago09.pdf

Rosabal, G. (2008). Estilos de aprendizaje y educación instrumental. Revista Electrónica "La Retreta”. Año I No.2. San José, Costa Rica. Recuperado de http://laretreta.net/0102/articulos/ estilosdeaprendizaje.html

Rosabal, G. (2009) Educación Musical en Costa Rica y Finlandia: Una discusión y comparación de contextos y objetivos. The Finish Journal of Music Education, Vol. 12 No. 2. Helsinki, Finlandia.

Rosabal, G. (2010) La Educación Musical en la escuela. Ensayo para la capacitación en línea de educadores de Iberoamérica. Módulos de Educación Artística del Centro de Altos Estudios Universitarios: Organización de los Estados Americanos.

Vargas, M. (2004) De las fanfarrias a las salas de concierto. San José, Costa Rica: Editorial de la Universidad de Costa Rica. 